\title{
HI 21 CM EMISSION LINE STUDY OF SOUTHERN GALACTIC SUPERNOVA REMNANTS
}

\author{
Bon-Chul Koo ${ }^{1}$, Ji-hyun Kang ${ }^{1}$, And N. M. MCClure-Griffiths ${ }^{2}$ \\ ${ }^{1}$ Astronomy Program, SEES, Seoul National University, Seoul 151-742, Korea; \\ E-mail: koo@astrohi.snu.ac.kr, kjh@astro.snu.ac.kr \\ ${ }^{2}$ ATNF-CSIRO, PO Box 76, Epping NSW 1710, Australia; \\ E-mail: Naomi.McClure-Griffiths@atnf.csiro.au \\ (Received May 19, 2004; Accepted June 10, 2004)
}

\begin{abstract}
We have searched for Hi $21 \mathrm{~cm}$ line emission from shocked atomic gas associated with southern supernova remnants (SNRs) using data from the Southern Galactic Plane Survey. Among the 97 sources studied, we have detected 10 SNRs with high-velocity HI emission confined to the SNR. The large velocity and the spatial confinement suggest that the emission is likely from the gas accelerated by the SN blast wave. We also detected 22 SNRs which show HI emission significantly brighter than the surrounding regions over a wide $\left(>10 \mathrm{~km} \mathrm{~s}^{-1}\right)$ velocity interval. The association with these SNRs is less certain. We present the parameters and maps of the excess emission in these SNRs. We discuss in some detail the ten individual SNRs with associated high-velocity HI emission.
\end{abstract}

Key words : supernova remnants - radio lines: ISM

\section{INTRODUCTION}

The HI emission line at $21 \mathrm{~cm}$ is very useful for studying radiative shocks in old supernova remnants (SNRs). The line is emitted from cold atomic gas, which is formed when the shock becomes radiative. This occurs when the shock has swept up enough ( $\gtrsim 10^{19} \mathrm{~cm}^{-2}$ ) column density. By analyzing $\mathrm{HI}$ emission lines from old SNRs, we can derive some fundamental SNR parameters, such as the shock velocity, mass and kinetic energy of the shocked gas, age of the SNR, etc. In addition, the HI data reveal the distribution of shocked atomic gas, which can be compared with the distribution of ambient atomic and molecular gas to explore the details of the interaction of SNRs with the ambient interstellar medium (ISM). Understanding such details, and in particular for a reasonably large statistical sample, is crucial for understanding the supernova process itself and also the overall effects of supernovae on the ISM.

Observationally, however, it is difficult to detect the HI emission line from shocked atomic gas, because most known SNRs are located in the Galactic plane where the Galactic background HI emission causes severe contamination. Only when the shock velocity is very large (e.g., $\gtrsim 100 \mathrm{~km} \mathrm{~s}^{-1}$ ) is the emission clearly discernible from the background emission. In the 1980's, highvelocity $(\mathrm{HV})$ emission was detected toward SNRs IC 443, G78.2+2.1, CTB 80, CTB 109, and VRO 42.05.01 (DeNoyer 1978; Giovanelli \& Haynes 1979; Landecker, Roger, and Higgs 1980; Braun \& Strom 1986; Koo et al. 1990). A systematic search of SNRs was made by
Koo \& Heiles (1991; hereafter KH91) using the Hat Creek $25 \mathrm{~m}$ telescope $\left(\mathrm{FWHM}=36^{\prime}\right)$. They observed 103 northern SNRs using the Hat Creek $25 \mathrm{~m}$ telescope $\left(\mathrm{FWHM}=36^{\prime}\right)$, which were all of the known SNRs at $\delta \gtrsim-38.5^{\circ}$ at that time. They observed each SNR at 9 points in a cross pattern centered at its catalog position and searched for broad excess emission brighter than the surrounding region. The SNRs with excess emission are divided into 3 ranks, in which increasing number implies increasing reliability of the detected $\mathrm{HI}$ feature. Fourteen SNRs (excluding G117.4+5.0 which is no longer considered an SNR any more [Green 2001]) showed $\mathrm{HV}$ excess emission which is quite likely from the gas accelerated by the SNR shock and ranked 3. The classical source IC 443 was classified as rank 2 because of some confusing emission in the surrounding region. The velocity of $\mathrm{HI}$ gas ranged from 70 to $160 \mathrm{~km}$ $\mathrm{s}^{-1}$ with respect to the systemic velocities. Follow-up high resolution observations have been made for SNRs CTB 80, W44, and W51C, and confirmed the association (Koo et al. 1993; Koo \& Heiles 1995; Koo \& Moon 1997).

In this paper, we present the results of a systematic search for shock-accelerated HV HI gas toward 97 SNRs in the southern sky. The organization of the paper is as follows: In $\S 2$, we describe the data and the procedure for classifying SNRs. We divide the SNRs into three ranks in a way similar to KH91. In $\S 3$, we present the tables and maps summarizing the results. We have detected ten SNRs with HV Hi gas localized at the positions of SNRs and another 22 SNRs with some excess emission at the position of SNRs. In $\S 4$, we discuss ten individual SNRs, and, in $\S 5$, we summarize the paper.

Corresponding Author: B.-C. Koo 

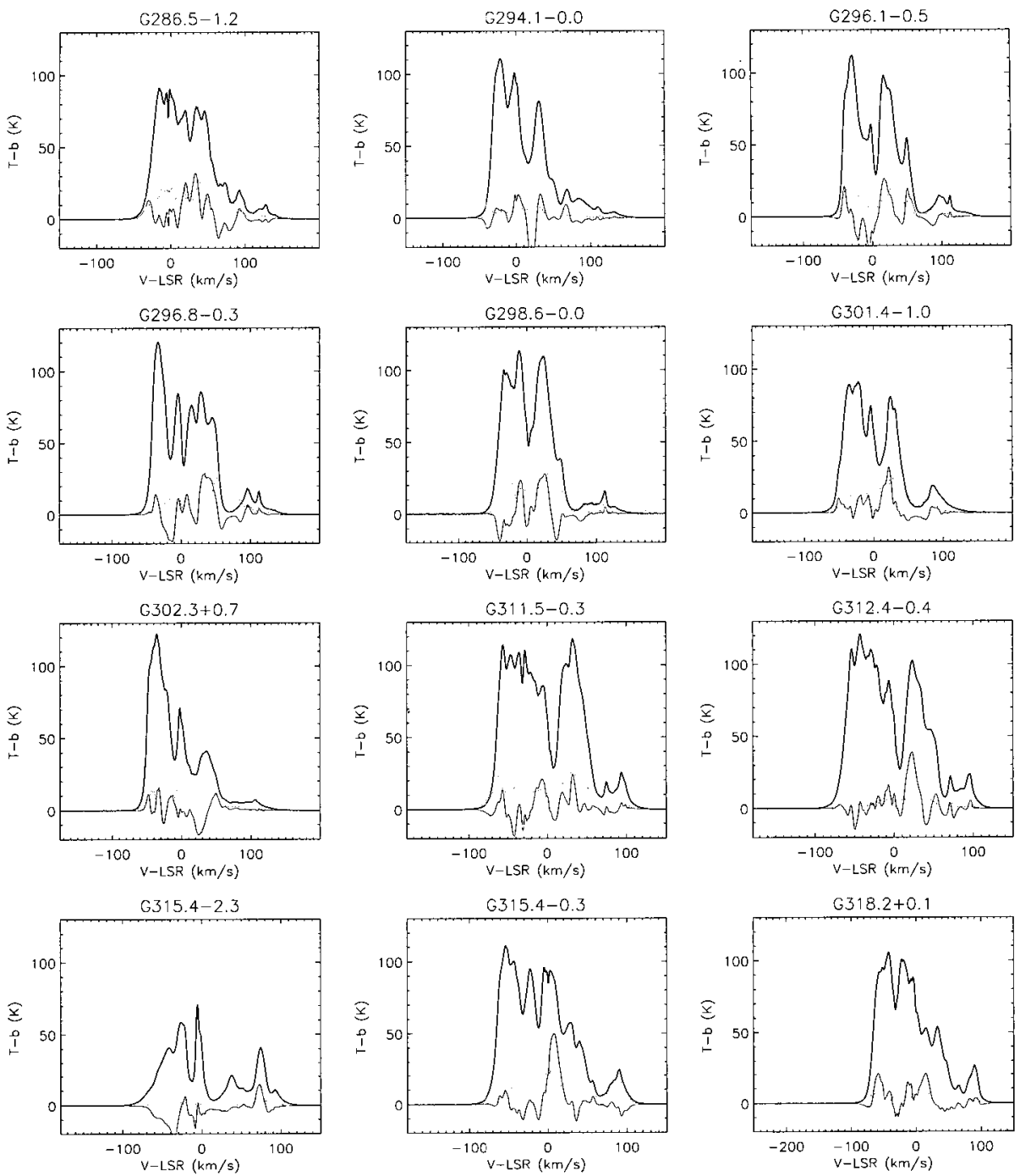

Fig. 1. - Spectra of rank 2 sources. The thick solid line is the average spectrum toward the SNR, the thin solid line is the difference spectrum of the SNR using the outer shell for the "OFF" spectrum, and the dotted line is the amplitude (1.5\%) of the background fluctuation obtained from the OFF region.

\section{DATA AND THE CLASSIFICATION}

We use Parkes data from the Southern Galactic Plane Survey (SGPS; McClure-Griffiths et al. 2001) for the search. The data covers $l=253^{\circ}-358^{\circ}$ and $|b| \lesssim 10^{\circ}$ with an angular resolution of $16^{\prime}$ (pixel size $\left.4^{\prime}\right)$ and a velocity resolution of $0.82 \mathrm{~km} \mathrm{~s}^{-1}$. The sensitivity ranges from 0.13 to $0.27 \mathrm{~K}$, but it is much better $(0.06-0.09 \mathrm{~K})$ in the latitude range $-1 .{ }^{\circ} 5 \lesssim b \lesssim 10^{\circ} 5$. With a sensitivity of $\sim 0.1 \mathrm{~K}(1.5 \sigma)$, the minimum detectable $\mathrm{HI}$ mass within a $10 \mathrm{~km} \mathrm{~s}^{-1}$ velocity interval is $\sim 0.4 d_{\mathrm{kpc}}{ }^{2} \mathrm{M}_{\odot}$, where $d_{\mathrm{kpc}}$ is the distance to the source in kpc.

There are 97 Galactic SNRs in Green's catalog (Green 2001) that lie in the longitude and latitude range covered by the SGPS data. We compare the average HI spectra of these SNRs to those of the sur- rounding regions, and look for excessive emission wider than $10 \mathrm{~km} \mathrm{~s}^{-1}$ and localized at the position of SNRs. Two circular/elliptical shells surrounding the SNR are used for the "OFF" spectra: one which is $8^{\prime}$ (half-beam width) thick and has an inner boundary at $8^{\prime}$ apart from the SNR boundary, and the other which is also $8^{\prime}$ thick and $8^{\prime}$ from the outer boundary of the former shell. We divide the SNRs into 3 ranks, where the increasing number implies increasing reliability of the detected $\mathrm{H}$ I feature:

Rank 2: SNRs which have an excessive emission over the outer shell by more than the 1.5 times of its rms fluctuation over a wide $\left(\geq 10 \mathrm{~km} \mathrm{~s}^{-1}\right)$ velocity interval.

Rank 3: SNRs which have an excessive emission over the inner and outer shells at extreme velocities:

All other sources are ranked 1. Figs. 1 and 2 show the 

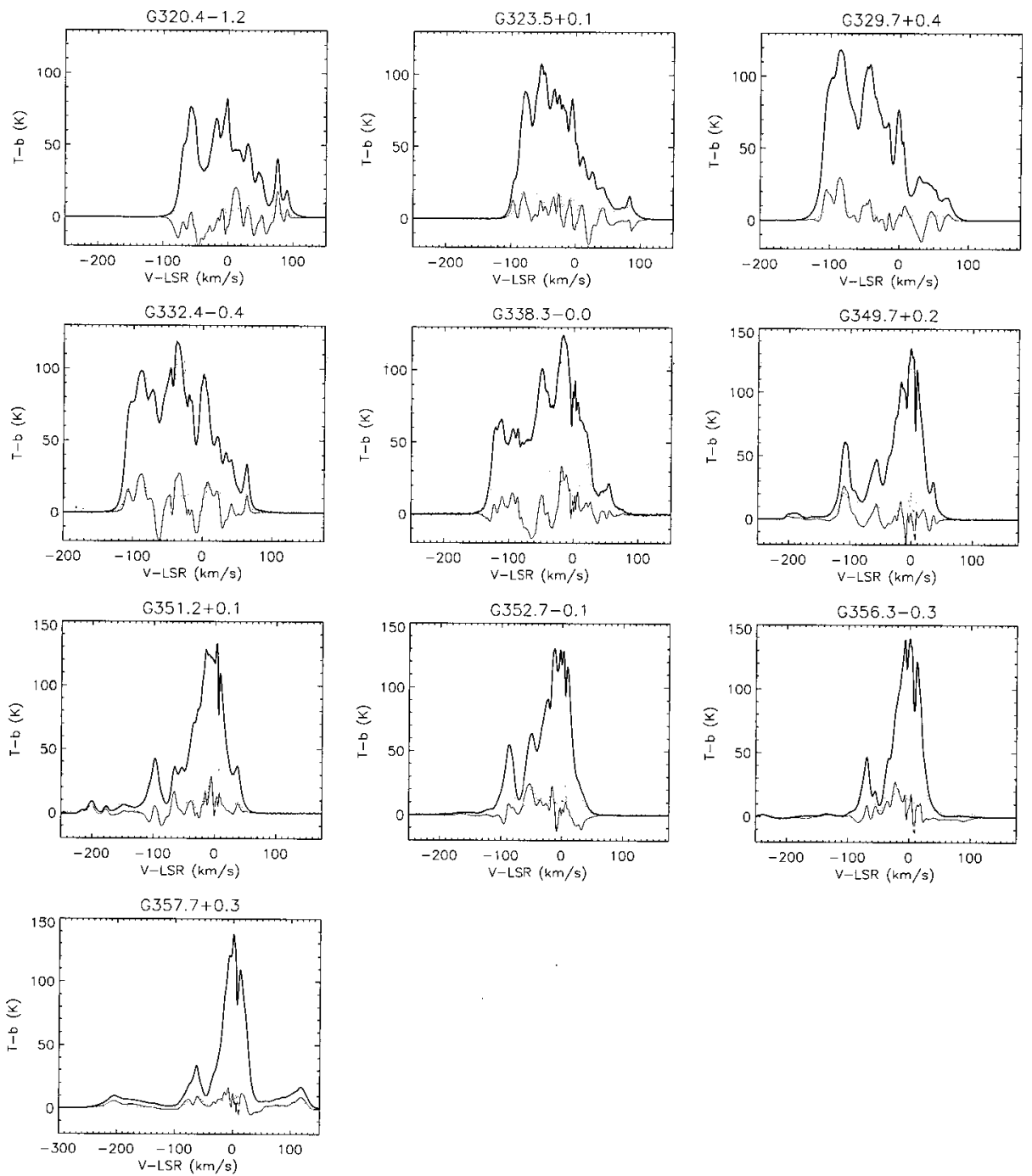

Fig. 1.- Continued.

spectra of ranks 2 and 3 sources, respectively.

Our classification is similar to the KH91's in principle, e.g., our ranks 2 and 3 correspond to their ranks 2 and 3 , respectively, while our rank 1 includes their ranks 0 and 1 . But, since we used a shell pattern instead of a cross pattern for the "OFF" spectra, the assigned ranks for individual SNRs could be different (see next section). SNRs ranked 3 have HV Hi gas confined to the SNR. Such gas is rare; thus, it is probable that the gas is really associated with the SNR. SNRs ranked 2 have excess HI gas confined to the position of SNRs, but not at an extreme velocity. Real association with the SNR is less certain, but the positional coincidence is suggestive.

\section{RESULTS}

The results of our survey are summarized in Table 1. Source parameters are from Green (2001). The size is the angular size in radio continuum; a single value is quoted for nearly circular remnants, and the product of two values, the major and minor axes, is quoted for elongated remnants. The type ' $\mathrm{S}$ ', ' $\mathrm{F}$ ', or 'C' represents SNRs with a 'shell', 'filled-centre', or 'composite' radio structure. The fluxes at $1 \mathrm{GHz}$ are also listed. The uncertain parameters are listed with a question mark. Of the 97 sources examined, 10 SNRs are ranked 3, 22 SNRs are ranked 2, and 65 SNRs are ranked 1. Nine SNRs had been observed by KH91: G261.9+5.5 (2), G348.5+0.1 (2), G348.7+0.3 (2), G349.7+0.2 (2), G351.2+0.1 (2), G352.7-0.1 (2), G355.9-2.5 (0), G357.7-0.1 (2), and G357.7+0.3 (2), where the numbers in parenthesis are the ranks assigned by KH91. We note that, for G261.9+5.5, G348.5+0.1, G348.7+0.3, and G357.7-0.1, we assign a rank of 1 , which KH91 assigned a rank of 2 . The disagreement is probably due to different observing patterns, e.g., shell versus cross. 

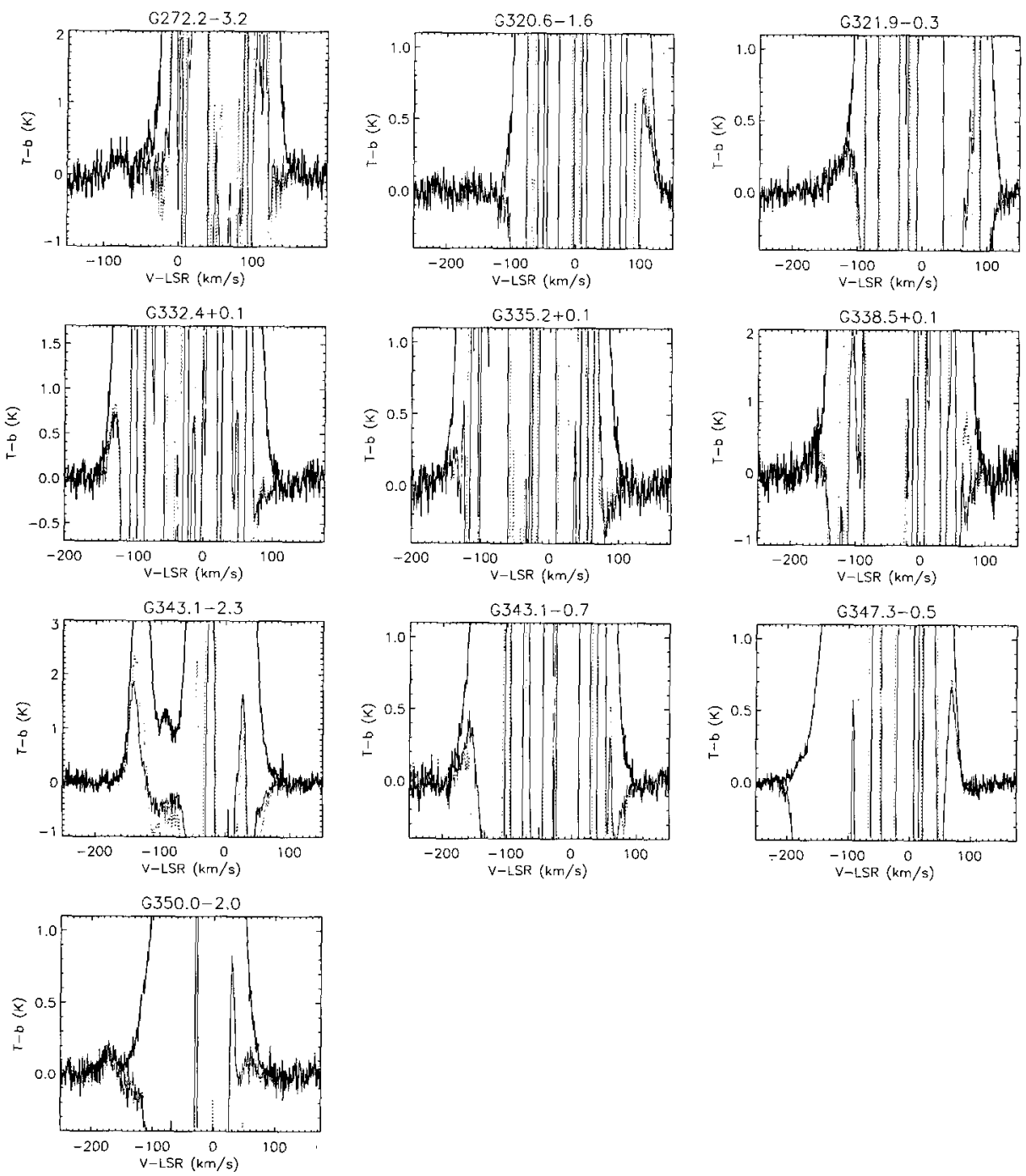

Fig. 2.- Spectra of rank 3 sources. The thick solid line is the average spectrum toward the SNR, while the thin solid and dotted lines are the difference spectra of the SNR using the inner and outer shells for the "OFF" spectrum, respectively.

In Table 2 we list, for rank 2 SNRs, the velocity intervals at which the SNR shows excessive emission brighter than $1.5 \sigma$ and wider than $10 \mathrm{~km} \mathrm{~s}^{-1}$ and the average integrated antenna temperature of the excess emission over the SNR area. The intensity of the excess emission ranges from 18 to $660 \mathrm{~K} \mathrm{~km} \mathrm{~s}^{-1}$, or 0.03 to $1.2 \times 10^{21} \mathrm{~cm}^{-2}$ in Hı column density (assuming that the emission is optically thin). Fig. 3 shows the maps of the excess emission where the dotted circles mark the positions and geometrical mean sizes of the SNRs. All rank 2 SNRs show at least some excess emission and we give a very short description of its morphology in Table 2. SNRs which show distinct excess emission confined to a small area within or near their boundaries are G286.5-1.2, G296.1-0.5, G301.4-1.0, and G302.3+0.7.

Table 3 summarizes the results of rank 3 SNRs. The velocity intervals are approximate intervals at which the SNRs show excessive emission compared to their surroundings. All sources, but two (G320.6-1.6 and G347.3-0.5) show excess emission at negative velocities. The intensities of the excess emission are much weaker than rank 2 SNRs, e.g., 2 to $34 \mathrm{~K} \mathrm{~km} \mathrm{~s}^{-1}$ or 0.3 to $6 \times 10^{19} \mathrm{~cm}^{-2}$. This may indicate that rank 3 SNRs have swept up less mass and therefore relatively younger than rank 2 SNRs, although some excess emission toward rank 2 SNRs might be due to random fluctuations of the background emission. Fig. 4 shows the maps of their excess emission. All sources show excess emission confined to them. The ones with particularly distinct excess emission are G272.2-3.2, G321.9-0.3, and G347.3-0.5. Individual sources are discussed in $\S 4$.

A characteristic of rank 3 SNRs is that most of them, 
TABLE 1

Summary of SuRvey Results

\begin{tabular}{|c|c|c|c|c|c|c|c|}
\hline $\begin{array}{c}\text { Galactic } \\
\text { Coordinates }\end{array}$ & Name(s) & $\begin{array}{r}\alpha(2000) \\
(\mathrm{h} \mathrm{m} \mathrm{s})\end{array}$ & $\begin{array}{c}\delta(2000) \\
\left({ }^{\circ} \quad '\right)\end{array}$ & $\begin{array}{c}\text { Size } \\
(\operatorname{arcmin})\end{array}$ & Type & $\begin{array}{c}\text { Flux at } \\
1 \mathrm{GHz} \\
(\mathrm{Jy})\end{array}$ & Rank \\
\hline $260.4-3.4$ & Puppis A, MSH 08-44 & 082210 & -4300 & $60 \times 50$ & $\mathrm{~S}$ & 130 & 1 \\
\hline $261.9+5.5$ & & 090420 & -3842 & $40 \times 30$ & $\mathrm{~S}$ & $10 ?$ & 1 \\
\hline $263.9-3.3$ & Vela(XYZ) & 083400 & -4550 & 255 & $\mathrm{C}$ & 1750 & 1 \\
\hline $266.2-1.2$ & $\ldots$ & 085200 & -4620 & 120 & $\mathrm{~S}$ & $50 ?$ & 1 \\
\hline $272.2-3.2$ & $\cdots$ & 090650 & -5202 & $15 ?$ & $\mathrm{~S} ?$ & 0.4 & 3 \\
\hline $279.0+1.1$ & & 095740 & -5315 & 95 & $\mathrm{~S}$ & $30 ?$ & 1 \\
\hline $284.3-1.8$ & MSH $10-53$ & 101815 & -5900 & $24 ?$ & $\mathrm{~S}$ & $11 ?$ & 1 \\
\hline $286.5-1.2$ & $\ldots$ & 103540 & -5942 & $26 \times 6$ & $\mathrm{~S} ?$ & $1.4 ?$ & 2 \\
\hline $289.7-0.3$ & $\ldots$ & 110115 & -6018 & $18 \times 14$ & $\mathrm{~S}$ & 6.2 & 1 \\
\hline $290.1-0.8$ & MSH $11-61 \mathrm{~A}$ & 110305 & -6056 & $19 \times 14$ & $\mathrm{~S}$ & 42 & 1 \\
\hline $291.0-0.1$ & (MSH 11-62) & 111154 & -6038 & $15 \times 13$ & $\mathrm{C}$ & 16 & 1 \\
\hline $292.0+1.8$ & MSH $11-54$ & 112436 & -5916 & $12 \times 8$ & $\mathrm{C}$ & 15 & 1 \\
\hline $292.2-0.5$ & & 111920 & -6128 & $20 \times 15$ & $\mathrm{~S}$ & $7 ?$ & 1 \\
\hline $293.8+0.6$ & $\ldots$ & 113500 & -6054 & 20 & $\mathrm{C}$ & $5 ?$ & 1 \\
\hline $294.1-0.0$ & $\cdots$ & 113610 & -6138 & 40 & $\mathrm{~S}$ & $>2 ?$ & 2 \\
\hline $296.1-0.5$ & $\cdots$ & 115110 & -6234 & $37 \times 25$ & $\mathrm{~S}$ & $8 ?$ & 2 \\
\hline $296.8-0.3$ & $1156-62$ & 115830 & -6235 & $20 \times 14$ & $\mathrm{~S}$ & 9 & 2 \\
\hline $298.5-0.3$ & & 121240 & -6252 & $5 ?$ & $?$ & $5 ?$ & 1 \\
\hline $298.6-0.0$ & $\ldots$ & 121341 & -6237 & $12 \times 9$ & $\mathrm{~S}$ & $5 ?$ & 2 \\
\hline $299.2-2.9$ & $\cdots$ & 121513 & -6530 & $18 \times 11$ & $\mathrm{~S}$ & $0.5 ?$ & 1 \\
\hline $299.6-0.5$ & $\cdots$ & 122145 & -6309 & 13 & $\mathrm{~S}$ & $1.0 ?$ & 1 \\
\hline $301.4-1.0$ & $\cdots$ & 123755 & -6349 & $37 \times 23$ & $\mathrm{~S}$ & $2.1 ?$ & 2 \\
\hline $302.3+0.7$ & $\cdots$ & 124555 & -6208 & 17 & $\mathrm{~S}$ & $5 ?$ & 2 \\
\hline $304.6+0.1$ & Kes 17 & 130559 & -6242 & 8 & $\mathrm{~S}$ & 14 & 1 \\
\hline $308.1-0.7$ & & 133737 & -6304 & 13 & $\mathrm{~S}$ & $1.2 ?$ & 1 \\
\hline $308.8-0.1$ & $\cdots$ & 134230 & -6223 & $30 \times 20 ?$ & $\mathrm{C} ?$ & $15 ?$ & 1 \\
\hline $309.2-0.6$ & $\cdots$ & 134631 & -6254 & $15 \times 12$ & $\mathrm{~S}$ & $7 ?$ & 1 \\
\hline $309.8+0.0$ & $\ldots$ & 135030 & -6205 & $25 \times 19$ & $\mathrm{~S}$ & 17 & 1 \\
\hline $310.6-0.3$ & Kes 20B & 135800 & -6209 & 8 & $\mathrm{~S}$ & $5 ?$ & 1 \\
\hline $310.8-0.4$ & Kes $20 \mathrm{~A}$ & 140000 & -6217 & 12 & $\mathrm{~S}$ & $6 ?$ & 1 \\
\hline $311.5-0.3$ & $\cdots$ & 140538 & -6158 & 5 & $\mathrm{~S}$ & $3 ?$ & 2 \\
\hline $312.4-0.4$ & $\cdots$ & 141300 & -6144 & 38 & $\mathrm{~S}$ & 45 & 2 \\
\hline $315.4-2.3$ & RCW 86, MSH 14-63 & 144300 & -6230 & 42 & $\mathrm{~S}$ & 49 & 2 \\
\hline $315.4-0.3$ & & 143555 & -6036 & $24 \times 13$ & $?$ & 8 & 2 \\
\hline $315.9-0.0$ & $\cdots$ & 143825 & -6011 & $25 \times 14$ & $\mathrm{~S}$ & $0.8 ?$ & 1 \\
\hline $316.3-0.0$ & (MSH 14-57) & 144130 & -6000 & $29 \times 14$ & $\mathrm{~S}$ & $20 ?$ & 1 \\
\hline $317.3-0.2$ & $\cdots$ & 144940 & -5946 & 11 & $\mathrm{~S}$ & $4.7 ?$ & 1 \\
\hline $318.2+0.1$ & $\cdots$ & 145450 & -5904 & $40 \times 35$ & $\mathrm{~S}$ & $>3.9 ?$ & 2 \\
\hline $318.9+0.4$ & $\ldots$ & 145830 & -5829 & $30 \times 14$ & $\mathrm{C}$ & $4 ?$ & 1 \\
\hline $320.4-1.2$ & MSH 15-52,RCW 89 & 151430 & -5908 & 35 & $\mathrm{C}$ & $60 ?$ & 2 \\
\hline $320.6-1.6$ & $\cdots$ & 151750 & -5916 & $60 \times 30$ & $\mathrm{~S}$ & $?$ & 3 \\
\hline $321.9-1.1$ & $\cdots$ & 152345 & -5813 & 28 & $\mathrm{~S}$ & $>3.4 ?$ & 1 \\
\hline $321.9-0.3$ & $\cdots$ & 152040 & -5734 & $31 \times 23$ & $\mathrm{~S}$ & 13 & 3 \\
\hline $322.5-0.1$ & $\cdots$ & 152323 & -5706 & 15 & $\mathrm{C}$ & 1.5 & 1 \\
\hline $323.5+0.1$ & $\cdots$ & 152842 & -5621 & 13 & $\mathrm{~S}$ & $3 ?$ & 2 \\
\hline
\end{tabular}


TABLe 1

- Continued

\begin{tabular}{|c|c|c|c|c|c|c|c|}
\hline $\begin{array}{c}\text { Galactic } \\
\text { Coordinates }\end{array}$ & Name(s) & $\begin{array}{r}\alpha(2000) \\
(\mathrm{h} \mathrm{m} \mathrm{s})\end{array}$ & $\begin{array}{c}\delta(2000) \\
\left(\begin{array}{c}0 \\
\end{array}\right)\end{array}$ & $\begin{array}{c}\text { Size } \\
\text { (arcmin) }\end{array}$ & Type & $\begin{array}{c}\text { Flux at } \\
1 \mathrm{GHz} \\
(\mathrm{Jy})\end{array}$ & Rank \\
\hline $326.3-1.8$ & MSH $15-56$ & 155300 & -5610 & 38 & $\mathrm{C}$ & 145 & 1 \\
\hline $327.1-1.1$ & $\cdots$ & 155425 & -5509 & 18 & $\mathrm{C}$ & $7 ?$ & 1 \\
\hline $327.4+0.4$ & Kes 27 & 154820 & -5349 & 21 & $\mathrm{~S}$ & $30 ?$ & 1 \\
\hline $327.4+1.0$ & $\ldots$ & 154648 & -5320 & 14 & $S$ & $1.9 ?$ & 1 \\
\hline $328.4+0.2$ & (MSH 15-57) & 155530 & -5317 & 5 & $\mathrm{~F}$ & 15 & 1 \\
\hline $329.7+0.4$ & $\cdots$ & 160120 & -5218 & $40 \times 33$ & $S$ & $>34 ?$ & 2 \\
\hline $330.2+1.0$ & $\cdots$ & 160106 & -5134 & 11 & S? & $5 ?$ & 1 \\
\hline $332.0+0.2$ & $\cdots$ & 161317 & -5053 & 12 & $\mathrm{~S}$ & $8 ?$ & 1 \\
\hline $332.4-0.4$ & RCW 103 & 161733 & -5102 & 10 & $\mathrm{~S}$ & 28 & 2 \\
\hline $332.4+0.1$ & MSH $16-51$, Kes 32 & 161517 & -5042 & 15 & $\mathrm{~S}$ & 26 & 3 \\
\hline $335.2+0.1$ & $\cdots$ & 162745 & -4847 & 21 & $\mathrm{~S}$ & 16 & 3 \\
\hline $336.7+0.5$ & $\cdots$ & 163211 & -4719 & $14 \times 10$ & $\mathrm{~S}$ & 6 & 1 \\
\hline $337.0-0.1$ & (CTB 33) & 163557 & -4736 & 1.5 & $\mathrm{~S}$ & 1.5 & 1 \\
\hline $337.2-0.7$ & & 163928 & -4751 & 6 & $S$ & $2 ?$ & 1 \\
\hline $337.3+1.0$ & Kes 40 & 163239 & -4636 & $15 \times 12$ & $\mathrm{~S}$ & 16 & 1 \\
\hline $337.8-0.1$ & Kes 41 & 163901 & -4659 & $9 \times 6$ & S & 18 & 1 \\
\hline $338.1+0.4$ & $\ldots$ & 163759 & -4624 & $15 ?$ & $\mathrm{~S}$ & $4 ?$ & 1 \\
\hline $338.3-0.0$ & $\cdots$ & 164100 & -4634 & 8 & $\mathrm{~S}$ & $7 ?$ & 2 \\
\hline $338.5+0.1$ & $\ldots$ & 164109 & -4619 & 9 & $?$ & $12 ?$ & 3 \\
\hline $340.4+0.4$ & $\cdots$ & 164631 & -4439 & $10 \times 7$ & $\mathrm{~S}$ & 5 & 1 \\
\hline $340.6+0.3$ & $\ldots$ & 164741 & -4434 & 6 & $\mathrm{~S}$ & $5 ?$ & 1 \\
\hline $341.2+0.9$ & $\ldots$ & 164735 & -4347 & $16 \times 22$ & $\mathrm{C}$ & $1.5 ?$ & 1 \\
\hline $341.9-0.3$ & $\cdots$ & 165501 & -4401 & 7 & $\mathrm{~S}$ & 2.5 & 1 \\
\hline $342.0-0.2$ & $\cdots$ & 165450 & -4353 & $12 \times 9$ & $\mathrm{~S}$ & $3.5 ?$ & 1 \\
\hline $342.1+0.9$ & $\cdots$ & 165043 & -4304 & $10 \times 9$ & $\mathrm{~S}$ & $0.5 ?$ & 1 \\
\hline $343.0-6.0$ & $\cdots$ & 172500 & -4630 & 250 & $\mathrm{~S}$ & $?$ & 1 \\
\hline $343.1-2.3$ & $\cdots$ & 170800 & -4416 & $32 ?$ & $\mathrm{C} ?$ & $8 ?$ & 3 \\
\hline $343.1-0.7$ & $\cdots$ & 170025 & -4314 & $27 \times 21$ & $\mathrm{~S}$ & 7.8 & 3 \\
\hline $344.7-0.1$ & $\cdots$ & 170351 & -4142 & 10 & $\mathrm{C} ?$ & $2.5 ?$ & 1 \\
\hline $345.7-0.2$ & $\ldots$ & 170720 & -4053 & 6 & $S$ & $0.6 ?$ & 1 \\
\hline $346.6-0.2$ & $\cdots$ & 171019 & -4011 & 8 & $\mathrm{~S}$ & $8 ?$ & 1 \\
\hline $347.3-0.5$ & $\ldots$ & 171350 & -3945 & $65 \times 55$ & $\mathrm{~S} ?$ & $?$ & 3 \\
\hline $348.5-0.0$ & $\ldots$ & 171526 & -3828 & $10 ?$ & $\mathrm{~S} ?$ & $10 ?$ & 1 \\
\hline $348.5+0.1$ & CTB 37A & 171406 & -3832 & 15 & $\mathrm{~S}$ & 72 & 1 \\
\hline $348.7+0.3$ & CTB 37B & 171355 & -3811 & $17 ?$ & $\mathrm{~S}$ & 26 & 1 \\
\hline $349.2-0.1$ & $\cdots$ & 171715 & -3804 & $9 \times 6$ & $\mathrm{~S}$ & $1.4 ?$ & 1 \\
\hline $349.7+0.2$ & $\cdots$ & 171759 & -3726 & $2.5 \times 2$ & $\mathrm{~S}$ & 20 & 2 \\
\hline $350.0-2.0$ & $\cdots$ & 172750 & -3832 & 45 & $\mathrm{~S}$ & 26 & 3 \\
\hline $351.2+0.1$ & $\ldots$ & 172227 & -3611 & 7 & $\mathrm{C} ?$ & $5 ?$ & 2 \\
\hline $351.7+0.8$ & $\cdots$ & 172100 & -3527 & $18 \times 14$ & $\mathrm{~S}$ & $10 ?$ & 1 \\
\hline $351.9-0.9$ & $\cdots$ & 172852 & -3616 & $12 \times 9$ & $\mathrm{~S}$ & $1.8 ?$ & 1 \\
\hline $352.7-0.1$ & $\cdots$ & 172740 & -3507 & $8 \times 6$ & $\mathrm{~S}$ & 4 & 2 \\
\hline $353.9-2.0$ & $\cdots$ & 173855 & -3511 & 13 & $\mathrm{~S}$ & $1 ?$ & 1 \\
\hline $354.1+0.1$ & $\cdots$ & 173028 & -3346 & $15 \times 3 ?$ & $\mathrm{C} ?$ & $?$ & 1 \\
\hline $354.8-0.8$ & $\cdots$ & 173600 & -3342 & 19 & $\mathrm{~S}$ & $2.8 ?$ & 1 \\
\hline
\end{tabular}


TABLE 1

- Continued

\begin{tabular}{cccccccc}
\hline \hline $\begin{array}{c}\text { Galactic } \\
\text { Coordinates }\end{array}$ & Name(s) & $\begin{array}{c}\alpha(2000) \\
(\mathrm{h} \mathrm{m} \mathrm{s})\end{array}$ & $\begin{array}{c}\delta(2000) \\
\left({ }^{\circ}{ }^{\prime}\right)\end{array}$ & $\begin{array}{c}\text { Size } \\
\text { (arcmin) }\end{array}$ & Type & $\begin{array}{c}\text { Flux at } \\
\text { 1 GHz } \\
(\mathrm{Jy})\end{array}$ & Rank \\
\hline $355.6-0.0$ & $\ldots$ & 173516 & -3238 & $8 \times 6$ & $\mathrm{~S}$ & $3 ?$ & 1 \\
$355.9-2.5$ & $\cdots$ & 174553 & -3343 & 13 & $\mathrm{~S}$ & 8 & 1 \\
$356.2+4.5$ & $\ldots$ & 171900 & -2940 & 25 & $\mathrm{~S}$ & 4 & 1 \\
$356.3-0.3$ & $\ldots$ & 173756 & -3216 & $11 \times 7$ & $\mathrm{~S}$ & $3 ?$ & 2 \\
$356.3-1.5$ & $\cdots$ & 174235 & -3252 & $20 \times 15$ & $\mathrm{~S}$ & $3 ?$ & 1 \\
$357.7-0.1$ & $\ldots$ & 174029 & -3058 & $8 \times 3$ & $?$ & 37 & 1 \\
$357.7+0.3$ & $\cdots$ & 173835 & -3044 & 24 & $\mathrm{~S}$ & 10 & 2 \\
\hline
\end{tabular}

TABLE 2

ExCESS EMISSIONS AT RANK 2 SNRS

\begin{tabular}{|c|c|c|c|c|c|c|c|}
\hline $\begin{array}{c}\text { Galactic } \\
\text { Coordinates }\end{array}$ & $\begin{array}{c}v_{\min }, v_{\max } \\
\left(\mathrm{km} \mathrm{s}^{-1}\right)\end{array}$ & $\begin{array}{c}\int_{\left(\mathrm{K} \mathrm{km} \mathrm{s}^{-1}\right)}^{\Delta T_{A} d v} \\
.\end{array}$ & Note & $\begin{array}{c}\text { Galactic } \\
\text { Coordinates }\end{array}$ & $\begin{array}{c}v_{\min }, v_{\max } \\
\left(\mathrm{km} \mathrm{s}^{-1}\right)\end{array}$ & $\begin{array}{c}\int{ }_{\left(\mathrm{K} \mathrm{km} \mathrm{s} \mathrm{s}^{-1}\right)} \\
\end{array}$ & Note \\
\hline $286.5-1.2$ & $-52.5,-28.6$ & $122.3 \pm 10.9$ & 1 & $320.4-1.2$ & $+5.2,+18.3$ & $217.7 \pm 11.1$ & 3 \\
\hline \multirow[t]{4}{*}{$294.1=0: 0$} & $+60.4,+71.1$ & $70: 9^{\prime} \pm 2.7$ & 1 & $323.5+0.1$ & $-110.3,-93.8$ & $94.3 \pm 7.8$ & 2 \\
\hline & $+29.0,+40.6$ & $130.0 \pm 12.6$ & 3 & $329.7+0.4$ & $+66.2,+78.5$ & $34.7 \pm 2.4$ & $1(?)$ \\
\hline & $-3.9,+10.9$ & $187.5 \pm 13.6$ & 3 & & $+5.2,+15.9$ & $75.4 \pm 6.9$ & 3 \\
\hline & $-29.5,-18.7$ & $69.2 \pm 8.3$ & 3 & & $-54.2,-38.5$ & $172.2 \pm 11.1$ & 3 \\
\hline \multirow[t]{2}{*}{$296.1-0.5$} & $\mp 48.0,+62.9$ & $174.8 \pm 2.0$ & 2 & & $-111.1,-76.5$ & $659.5 \pm 26.4$ & 3 \\
\hline & $+10.1,+23.3$ & $290.2 \pm 25.1$ & 2 & $332.4-0.4$ & $-40.2,-27.0$ & $306.6 \pm 28.1$ & 3 \\
\hline $296.8-0.3$ & $+35.7,+46.4$ & $286.9 \pm 30.7$ & 2 & $338.3-0.0$ & $-21.2,-7.2$ & $355.8 \pm 44.3$ & 3 \\
\hline $298.6-0.0$ & $+15.9,+28.3$ & $314.2 \pm 37.1$ & 3 & $349.7+0.2$ & $-115.2,-97.9$ & $489.8 \pm 60.6$ & 3 \\
\hline $301.4-1.0$ & $+11.8,+23.3$ & $250.4 \pm 25.9$ & 1 & $351.2+0.1$ & $-210.0,-196.8$ & $83.3 \pm 8.6$ & 3 \\
\hline $302.3+0.7$ & $+44.7,+56.3$ & $108.2 \pm 10.0$ & 2 & $352.7-0.1$ & $-61.6,-46.8$ & $413.5 \pm 50.6$ & 3 \\
\hline $311.5-0.3$ & $-15.4,-1.4$ & $232.9 \pm 25.0$ & 3 & $356.3-0.3$ & $-244.6,-233.9$ & $18.2 \pm 3.4$ & 3 \\
\hline $312.4-0.4$ & $+12.6,+34.0$ & $532.8 \pm 19: 8$ & 3 & $357.7+0.3$ & $+104.7,+127.8$ & $166.2 \pm 25.1$ & 3 \\
\hline $315.4-2.3$ & $+66.2,+78.5$ & $116.1 \pm 5.0$ & $3(?)$ & & $+56.1,+74.2$ & $40.3 \pm 8.9$ & 3 \\
\hline $315.4-0.3$ & $-0.6,+17.5$ & $657.3 \pm 46.8$ & 3 & & $-17.3,-5.8$ & $153.6 \pm 23.3$ & 3 \\
\hline \multirow[t]{2}{*}{$318.2+0.1$} & $-2.2,+21.7$ & $314.3 \pm 14.5$ & 2 & & $-86.6,-74.2$ & $73.3 \pm 16.3$ & 3 \\
\hline & $-65.7,-49.2$ & $248.3 \pm 14.0$ & 3 & & $-244.0,-147.6$ & $267.2 \pm 15.5$ & 3 \\
\hline
\end{tabular}

NOTE:--.(1) Excess emission confined to a small area centered at SNR. (2) Excess emission confined to a small area neâr SNR b6undary. (3) Excess emission extended over a large area surrounding SNR. 

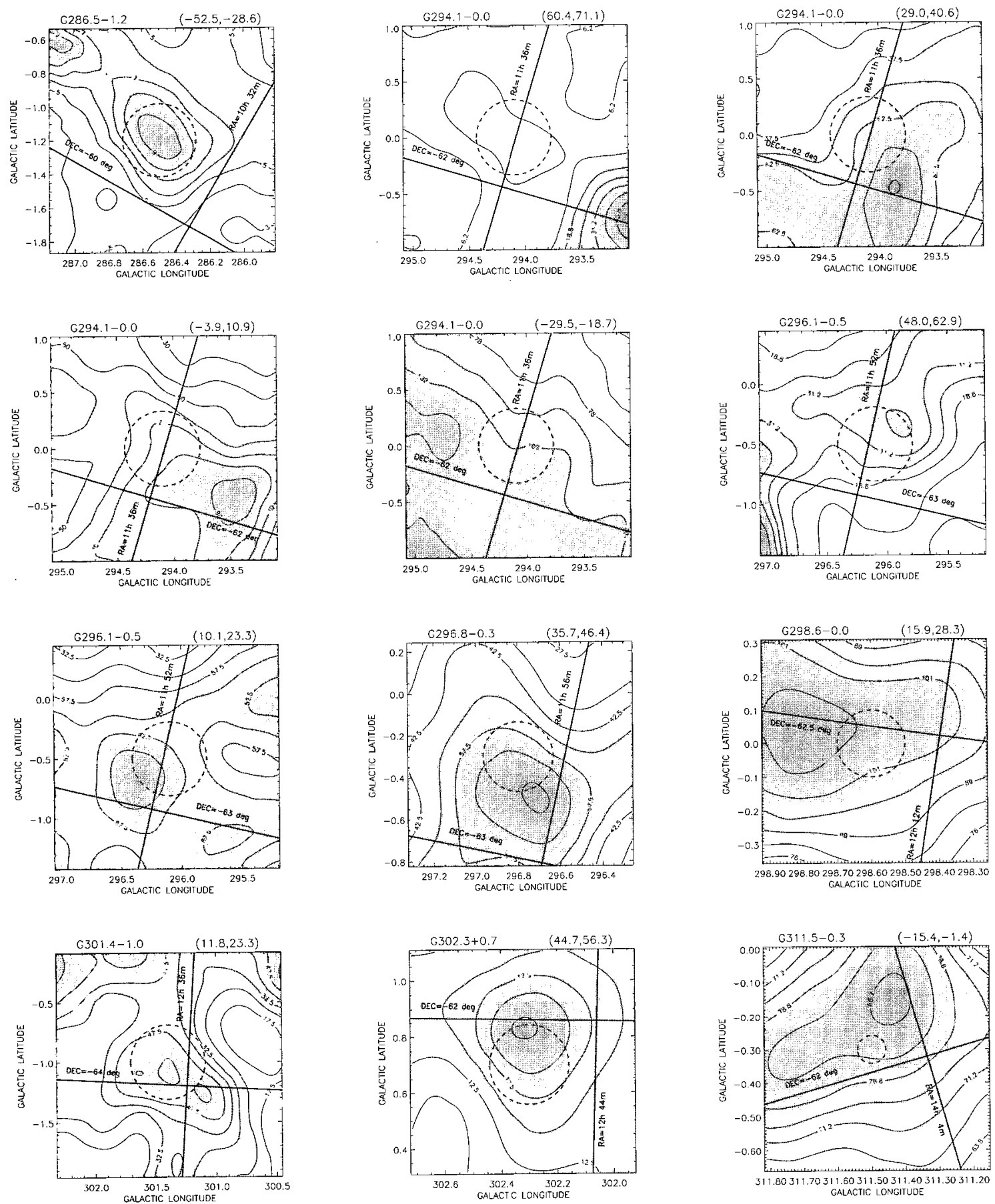

Fig. 3.- Contour maps of the excess emission of the rank-2 SNRs. The contours are labeled by average antenna temperature in $\mathrm{K}$. The grey scale plot distinguishes between valley and hill, and the higher integrated intensity is blacker. The dashed circles represent the locations and sizes of SNRs. The source name and the velocity range appears at the top of each picture. 

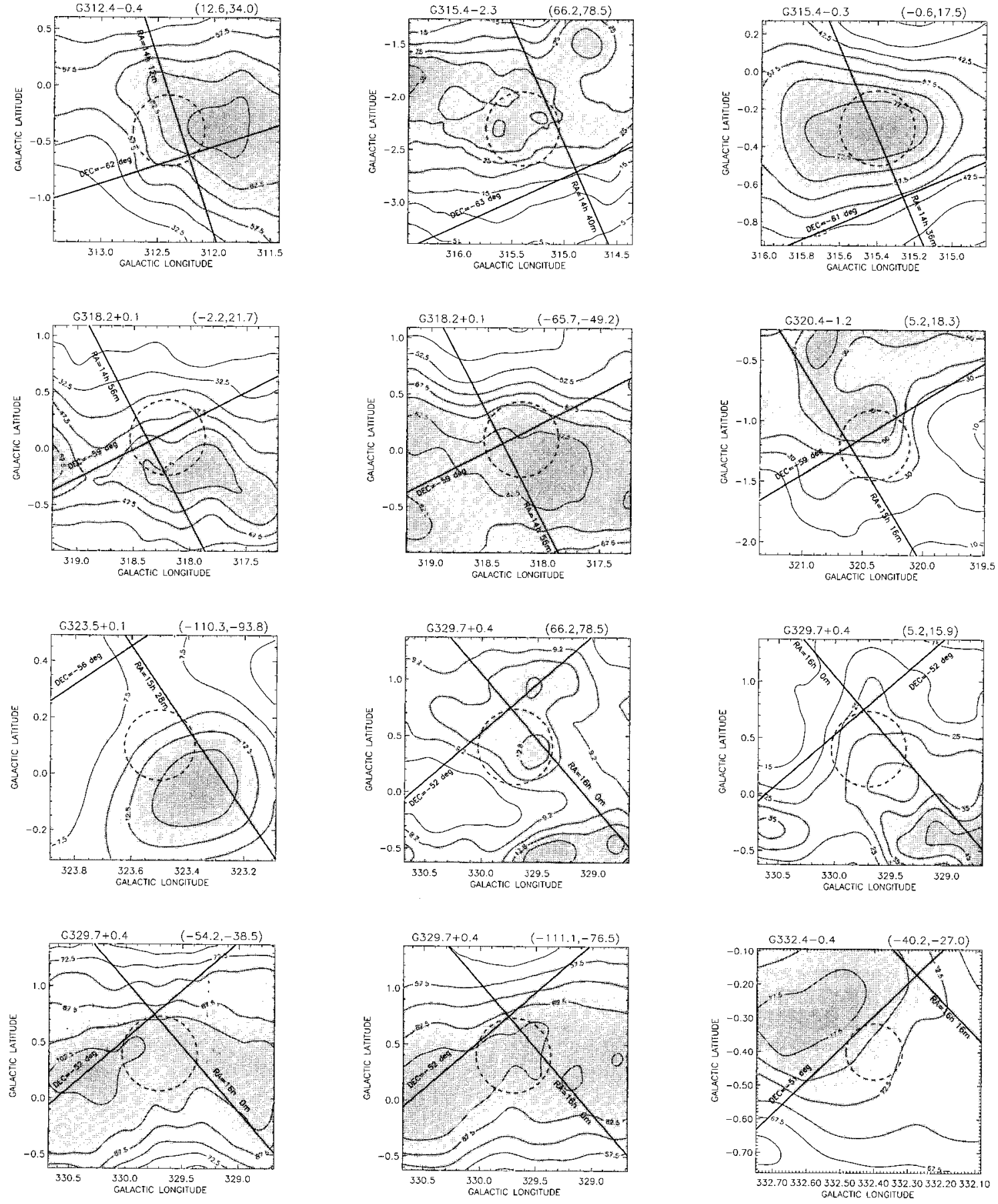

Fig. 3.- Continued. 

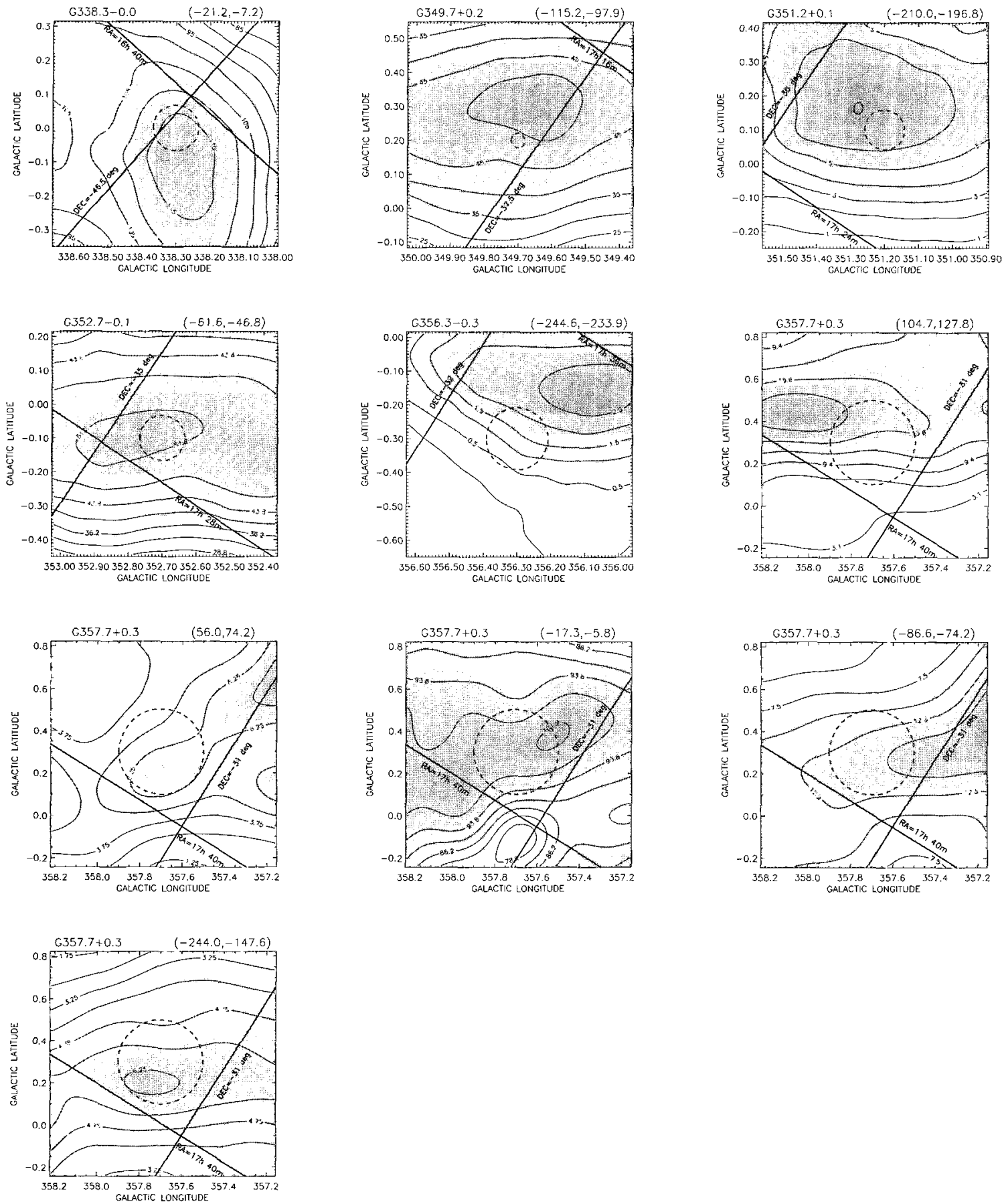

Fig. 3.- Continued. 

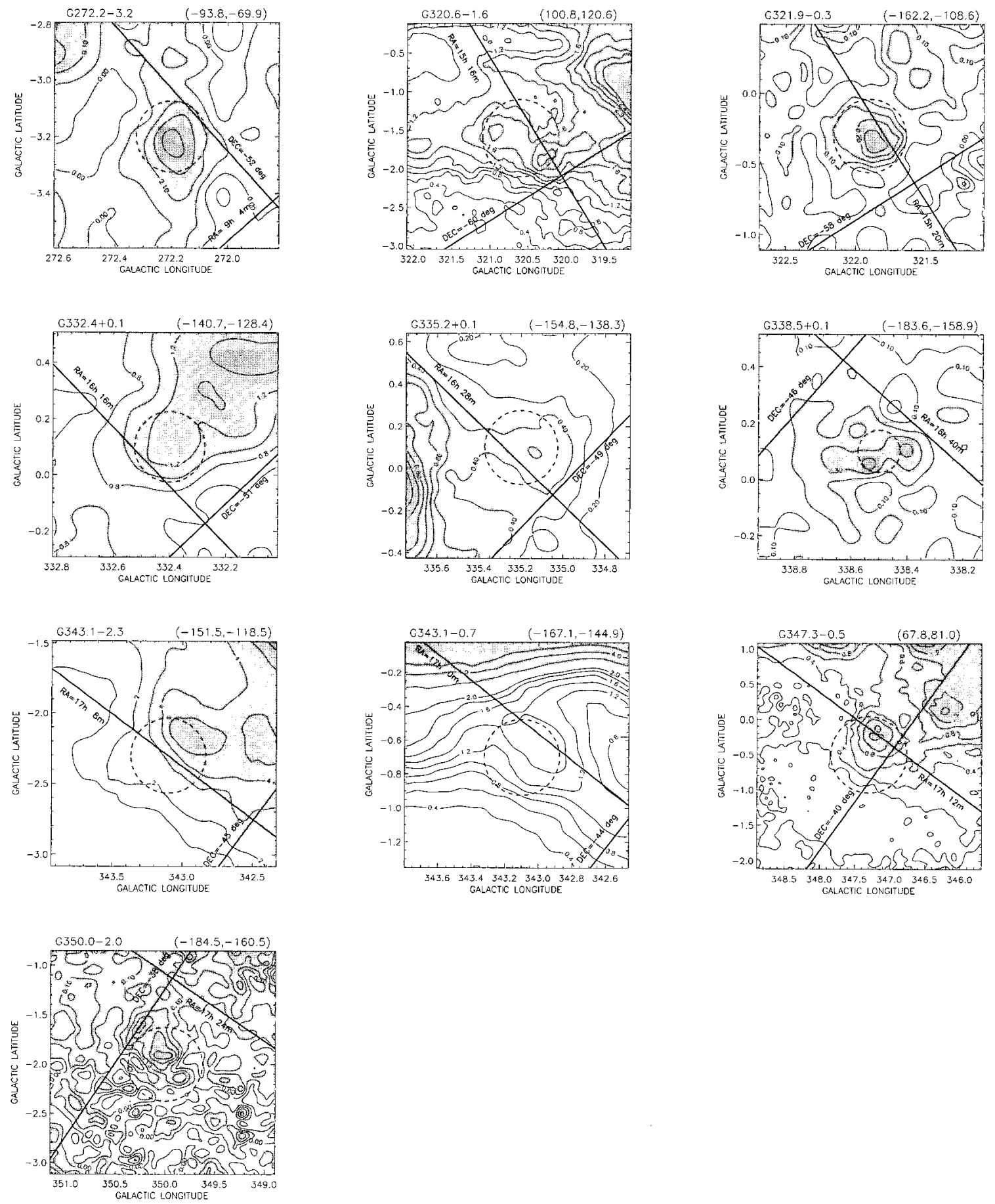

Fig. 4.- Same as Fig. 3, but for rank-3 SNRs. 
TABlE 3

High-Velocity Excess EMISSION AT RANK 3 SNRS

\begin{tabular}{cccccccc}
\hline $\begin{array}{c}\text { Galactic } \\
\text { Coordinates }\end{array}$ & $\begin{array}{c}v_{\min }, v_{\max } \\
\left(\mathrm{km} \mathrm{s}^{-1}\right)\end{array}$ & $\begin{array}{c}\int \Delta \bar{T}_{A} d v \\
\left(\mathrm{~K} \mathrm{~km} \mathrm{~s}^{-1}\right)\end{array}$ & Note & $\begin{array}{c}\text { Galactic } \\
\text { Coordinates }\end{array}$ & $\begin{array}{c}v_{\min }, v_{\max } \\
\left(\mathrm{km} \mathrm{s}^{-1}\right)\end{array}$ & $\begin{array}{c}\int \Delta \bar{T}_{A} d v \\
\left(\mathrm{~K} \mathrm{~km} \mathrm{~s}^{-1}\right)\end{array}$ & Note \\
\hline & & & & & & & \\
$272.2-3.2$ & $-93.8,-69.9$ & $4.6 \pm 0.6$ & 1 & $338.5+0.1$ & $-183.6,-158.9$ & $1.7 \pm 0.9$ & 2 \\
$320.6-1.6$ & $+100.8,+120.6$ & $10.4 \pm 0.7$ & 3 & $343.1-2.3$ & $-151.5,-118.5$ & $34.0 \pm 2.1$ & 3 \\
$321.9-0.3$ & $-162.2,-108.6$ & $8.7 \pm 0.5$ & 1 & $343.1-0.7$ & $-167.1,-144.9$ & $5.6 \pm 1.7$ & 3 \\
$332.4+0.1$ & $-140.7,-128.4$ & $9.6 \pm 2.6$ & 3 & $347.3-0.5$ & $+67.8,+81.0$ & $9.1 \pm 0.2$ & 1 \\
$335.2+0.1$ & $-154.8,-138.3$ & $3.0 \pm 1.1$ & 3 & $350.0-2.0$ & $-184.5,-160.5$ & $2.4 \pm 0.1$ & 2 \\
\hline
\end{tabular}

Note.-(1) Excess emission confined to SNR. (2) Excess emission scattered over an area surrounding SNR. Not enough sensitivity. (3) Excess emission in confusing area.

8 out of 10 , show excess emission at negative velocities. We note that they are all in the fourth quadrant at $l \gtrsim 320^{\circ}$. According to KH91, the ones in the first quadrant, 12 out of 14 , show excess emission at positive velocities. This highly contrasting tendency is consistent with what we would expect from the Galactic rotation: Suppose that there is an SNR which has a spherically symmetric, expanding HI shell in the first quadrant in the inner Galaxy. Along the direction toward the SNR, there is a strong Galactic background HI emission over a wide velocity interval, from negative to positive velocities. Now since the LSR velocity of the SNR is positive, only the receding portion of the shell will be detectable. For those in the fourth quadrant, it will be the opposite. Therefore, the trend seems to suggest that most of the Hi features in Table 3 are produced by sources in the inner Galaxy, probably the SNRs.

Fig. 5 shows the longitude distribution of SNRs in different ranks. The total number of observed SNRs is 189 including 92 SNRs observed by KH91. * For comparison, the total number of SNRs compiled by Green in 2001 is 231 (Green 2001). The longitude distribution of SNRs is slightly asymmetric with a relatively small number of SNRs between $l=240^{\circ}$ and $290^{\circ}$. Out of 189 SNRs, 29 SNRs are classified as rank 3, 39 SNRs as rank 2 , and the rest as rank 1 . Fig. 5 shows that $90 \%$ of rank-2 SNRs are between $l=290^{\circ}$ and $50^{\circ}$, while it is $73 \%$ for all the observed SNRs. This crowding of rank 2 SNRs in the inner Galaxy was noted by KH91 who attributed it to the confusion, i.e., the long path length gives a high probability for the random fluctuations of the background emissions to produce rank-2

* KH91 observed 103 northern SNRs, nine of which are included in this study. Excluding these nine SNRs and G112.0+1.2 and G117.4+5.0 which are no longer thought be SNRs, we are left with 92 SNRs. sources. There is no such crowding of rank 3 SNRs.

\section{DISCUSSION OF INDIVIDUAL SUERPNOVA REMNANTS WITH HIGH- VELOCITY HI GAS}

In the following we discuss the individual rank 3 SNRs that are given listed in Table 3. Three SNRs, G272.2-3.2, G321.9-0.3, and G347.3-0.5, are discussed in detail, while the other SNRs are discussed very briefly.

\section{(a) G272.2-3.2}

G272.2-3.2 was discovered in the ROSAT All-Sky Survey (Greiner \& Egger 1993) and the follow-up Xray and radio observations confirmed that it was a SNR (Greiner, Egger, \& Aschenbach 1994; Duncan et al. 1997). The SNR appears centre-filled both in radio and in $\mathrm{X}$-rays and is almost circular with a diameter of about $15^{\prime}$ (Duncan et al. 1997; Harrus et al. 2001). The spectral index $(0.55 \pm 0.15)$ of the radio emission is steeper than plerions and the $\mathrm{X}$-ray spectrum is thermal. In a detailed X-ray study, Harrus et al. (2001) found that the X-ray emission from the SNR is best described by a nonequilibrium ionization model with a temperature around $0.7 \mathrm{keV}$, ionization timescale of $3200 \mathrm{~cm}^{-3} \mathrm{yr}$, and absorbing column density of $1.1 \times 10^{22} \mathrm{~cm}^{-2}$. Those authors also discovered an infrared shell surrounding the western boundary of the SNR in the IRAS $60 / 100 \mu \mathrm{m}$ surface brightness map. The distance to the SNR is uncertain. Greiner, Egger, \& Aschenbach (1994) determined a distance of $1.8_{-0.8}^{+1.4} \mathrm{kpc}$ by comparing their X-ray absorbing columns $\left(4.6 \pm 3 \times 10^{21} \mathrm{~cm}^{-2}\right)$ to a mean interstellar absorbing columns $\left(2.5 \times 10^{21} \mathrm{~cm}^{-2} \mathrm{kpc}^{-1}\right)$. As they pointed out, this distance would imply an initial SN explosion energy of $2.5 \times 10^{49}$ erg based on the Sedov model, which is uncomfortably small. On the other 
hand, Harrus et al. (2001) derived an upper limit of $10 \mathrm{kpc}$ by comparing their X-ray absorbing columns $\left(1.12 \pm 0.02 \times 10^{22} \mathrm{~cm}^{-2}\right)$ to the interstellar absorbing columns $\left(1.2 \times 10^{21} \mathrm{~cm}^{-2} \mathrm{kpc}^{-1}\right)$ derived by Lucke (1978) toward this direction, and adopted a distance of $5 \mathrm{kpc}$.

The excess Hi emission toward G272.2-3.2 appears as a faint $(\lesssim 0.3 \mathrm{~K})$ bump at $v_{\mathrm{LSR}}=-94$ and $-70 \mathrm{~km}$ $\mathrm{s}^{-1}$ (Fig. 2). Fig. 6 shows the contour map of the integrated intensity overlaid on the X-ray image of G272.2-3.2. The HI emission peaks at the western portion of the remnant where the infrared shell has been detected. The X-rays are enhanced at the western edge of the SNR and the SNR boundary is somewhat flattened at this location. All these observations seem to indicate that G272.2-3.2 has been interacting with a dense medium in the west, but the almost circular shape of the SNR suggests that the interaction might have started rather recently. Also, the detection of the HV HI gas suggests that the SNR shock in this region is radiative. At a distance of $5 \mathrm{kpc}$, the radius $\left(7.5^{\prime}\right)$ of the SNR is $11 \mathrm{pc}$ and its systematic velocity is $27 \mathrm{~km}$ $\mathrm{s}^{-1}$ (assuming a flat rotation curve with $R_{\odot}=8.5 \mathrm{kpc}$ and $v_{\odot}=220 \mathrm{~km} \mathrm{~s}^{-1}$ ). The maximum velocity of the Hi gas that we detected corresponds to an expansion velocity of $v_{s} \simeq 120 \mathrm{~km} \mathrm{~s}^{-1}$ with respect to the SNR.

\section{(b) G321.9-0.3}

G321.9-0.3 is a shell-type SNR with a bright western shell. Kesteven \& Caswell (1987) classified the SNR as one of the barrel-shaped SNRs, which are the SNRs with two symmetric bright limbs with little or no emissivity in the end-caps. No reliable distance estimates are available. Barring any other distance indicators, one can use the empirical $\Sigma-D$ relation to estimate the distance to within about a factor of two. Using the relationship given by Case \& Bhattacharya (1998), we estimate a distance of $5.5 \mathrm{kpc}$ (cf. Clark, Caswell, \& Green 1975), but note that this is very uncertain. At this distance, the major and minor axis of the SNR become $50 \mathrm{pc}$ and $37 \mathrm{pc}$, respectively. G321.9-0.3 is particularly interesting because there is a X-ray binary, Circinus X-1, located $10^{\prime}$ north of the northern rim of the SNR. It has been proposed that Circinus X-1 is a runaway binary ejected from the SN explosion (Clark, Parkinson, \& Caswell 1975; Stewart et al. 1993). The minimum distance to Cir X-1 from Hi absorption experiment is $6.7 \mathrm{kpc}$ (Goss \& Mebold 1977).

G321.9-0.3 is a prototype of rank 3 sources. It has a broad $\left(\sim 50 \mathrm{~km} \mathrm{~s}^{-1}\right)$ wing clearly confined to the SNR. Fig. 7 shows the contour map of the integrated intensity between $v_{\mathrm{LSR}}=-162$ and $-109 \mathrm{~km} \mathrm{~s}^{-1}$ overlaid on the radio continuum image of the SNR. The Hı emission peaks at the very center of the remnant. If the distance to the SNR is $5.5 \mathrm{kpc}$, its systemic velocity would be $-78 \mathrm{~km} \mathrm{~s}^{-1}$ and the maximum velocity of the Hi gas corresponds to an expansion velocity of $\sim 80 \mathrm{~km} \mathrm{~s}^{-1}$.

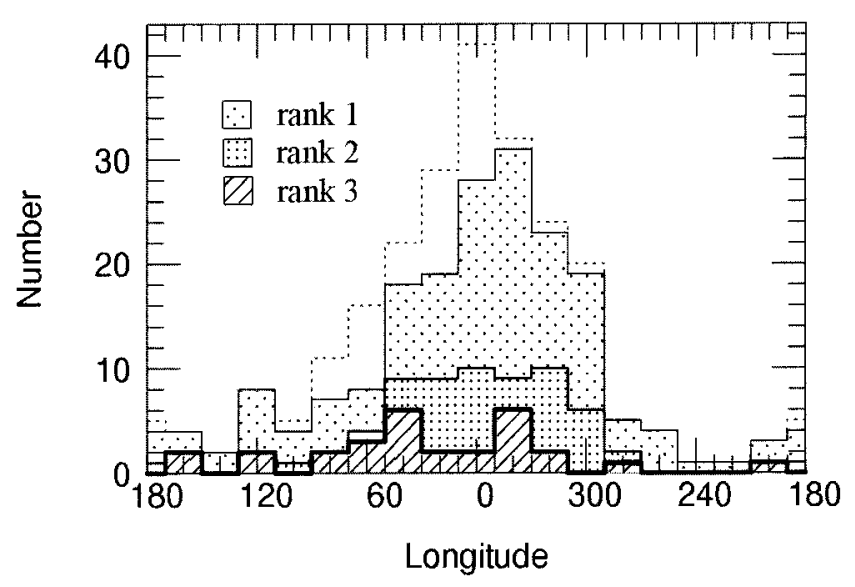

Fig. 5.- Distribution of SNRs in Galactic longitude. The histograms in solid line show the distribution of ranks 3,2 , and 1 SNRs from bottom to top in cumulative way, e.g., the sum under the top histogram is the total number (189) of observed SNRs. The top histogram in dotted line shows the distribution of all 231 SNRs in Green's catalog (Green 2001).

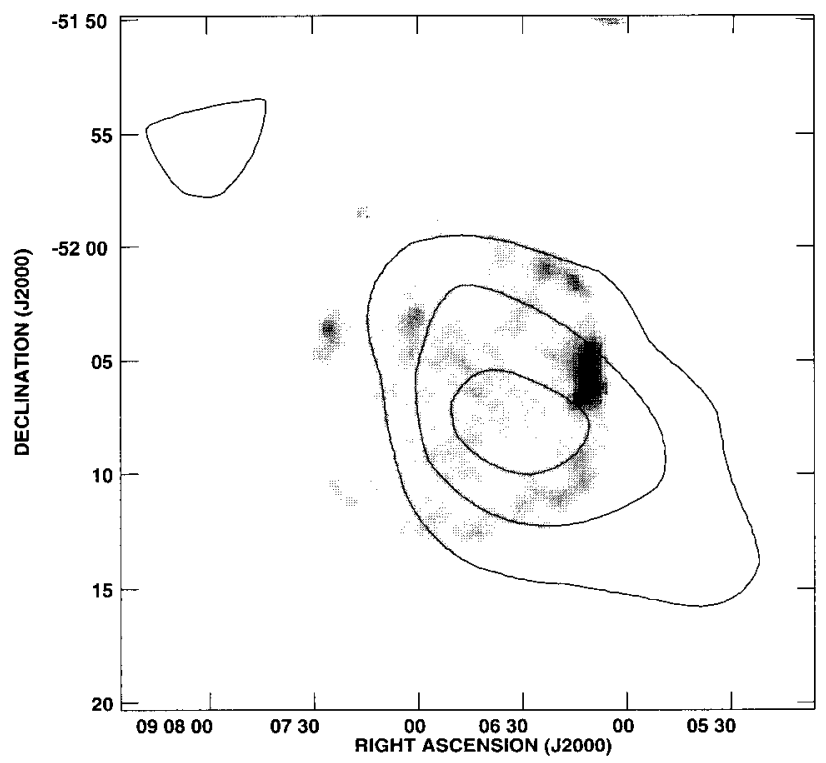

Fig. 6.- Contour map of the HI excess emission overlaid on the ROSAT X-ray image of G272.2-3.2. The contour levels are $0.1,0.15$, and $0.2 \mathrm{~K}$ in average antenna temperature over $v_{\mathrm{LSR}}=-94$ to $-70 \mathrm{~km} \mathrm{~s}^{-1}$.

The characteristic age of the remnant, assuming that it is in a pressure-driven snowplow phase, is $\sim 8 \times 10^{4} \mathrm{yr}$. If Cir X-1 was ejected from the center of G321.9-0.3, then, the current distance $\left(25^{\prime}=40 \mathrm{pc}\right)$ implies a transverse speed of $490 \mathrm{~km} \mathrm{~s}^{-1}$. 


\section{(c) G347.3-0.5}

G347.3-0.5 (RX J1713.7-3946) is bright X-ray SNR discovered in the ROSAT All-Sky Survey (Pfeffermann \& Aschenbach 1996). It has a bright western shell and an unidentified point source at the center. The X-ray spectrum of the diffuse emission is non-thermal, which indicates the presence of high-energy electrons accelerated by the SNR shock (Koyama et al. 1997; Slane et al. 1999; Uchiyama, Aharonian, \& Takahashi 2003; Lazendic et al. 2004). TeV gamma-ray emission has been detected toward the X-ray bright western shell, which is attributed to the inverse Compton scattering of the Cosmic Microwave Background Radiation by high-energy electrons (Muraishi et al. 2000) or pion decay (Enomoto et al. 2002). At radio wavelengths, the SNR is generally very faint with a few relatively bright arc-like features along the west rim (Slane et al. 1999). The distance to the SNR is controversial: Koyama et al. (1997) adopted $1 \mathrm{kpc}$ by comparing the $\mathrm{X}$-ray derived absorbing columns $\left(6 \times 10^{21} \mathrm{~cm}^{-2}\right)$ to the average total $\left(6 \times 10^{22} \mathrm{~cm}^{-2}\right)$ columns in the direction of the Galactic center. This gives a radius of $9 \mathrm{pc}$ and suggests that the SNR is young, possibly the remnant of the historical SN AD 393 (Wang et al. 1997). But Slane et al. (1999) pointed out that the total column density toward the SNR direction $\left(1.2 \times 10^{22} \mathrm{~cm}^{-2}\right)$ is significantly lower than the average in this direction, so that the distance to the remnant must be considerably larger than $1 \mathrm{kpc}$. They further argued that the SNR is possibly associated with molecular clouds in the vicinity, which have central velocities of $-94 \mathrm{~km} \mathrm{~s}^{-1}$ and/or $-69 \mathrm{~km} \mathrm{~s}^{-1}$, and adopted $6 \mathrm{kpc}$. This gives the SNR radius of $60 \mathrm{pc}$ and suggests that the SNR old. Koo, Kang, \& McClure-Griffiths (2004) showed how the column density of hydrogen nuclei $\left(\mathrm{HI}+\mathrm{H}_{2}\right)$ varies with distance toward the SNR using a kinematic model of the Galaxy and showed that the accumulated column density becomes comparable to the X-ray absorbing columns at $\sim 1 \mathrm{kpc}$.

The excess $\mathrm{HI}$ emission toward G347.3-0.5 is detected between $v_{\mathrm{LSR}}=68$ and $81 \mathrm{~km} \mathrm{~s}^{-1}$ at the western rim where there is enhanced X-ray emission. Fig. 8 shows ROSAT X-ray image of the SNR with the total integrated $\mathrm{H}_{\mathrm{I}}$ intensity contours overlaid. At a distance of $1 \mathrm{kpc}$, the radius $\left(30^{\prime}\right)$ of the SNR is $9 \mathrm{pc}$ and the systematic velocity of the SNR would be $\sim-6 \mathrm{~km} \mathrm{~s}^{-1}$. The maximum velocity of the $\mathrm{HI}$ gas corresponds to an expansion velocity of $\sim 90 \mathrm{~km} \mathrm{~s}^{-1}$. The X-ray observations, however, showed that thermal X-rays from shocked gas are absent or at least very weak, which has been considered to be due to very tenuous ambient medium (Slane et al. 1999). The SNR, then, must be very young and the shock should be non-radiative. The HV Hi gas could still have been produced by SNR shocks propagating through ambient molecular clouds. Indeed there are molecular clouds surrounding the SNR at $v_{\mathrm{LSR}} \sim-10 \mathrm{~km} \mathrm{~s}^{-1}$, which have been suggested to be interacting with the SNR (Fukui et al. 2003). Al-

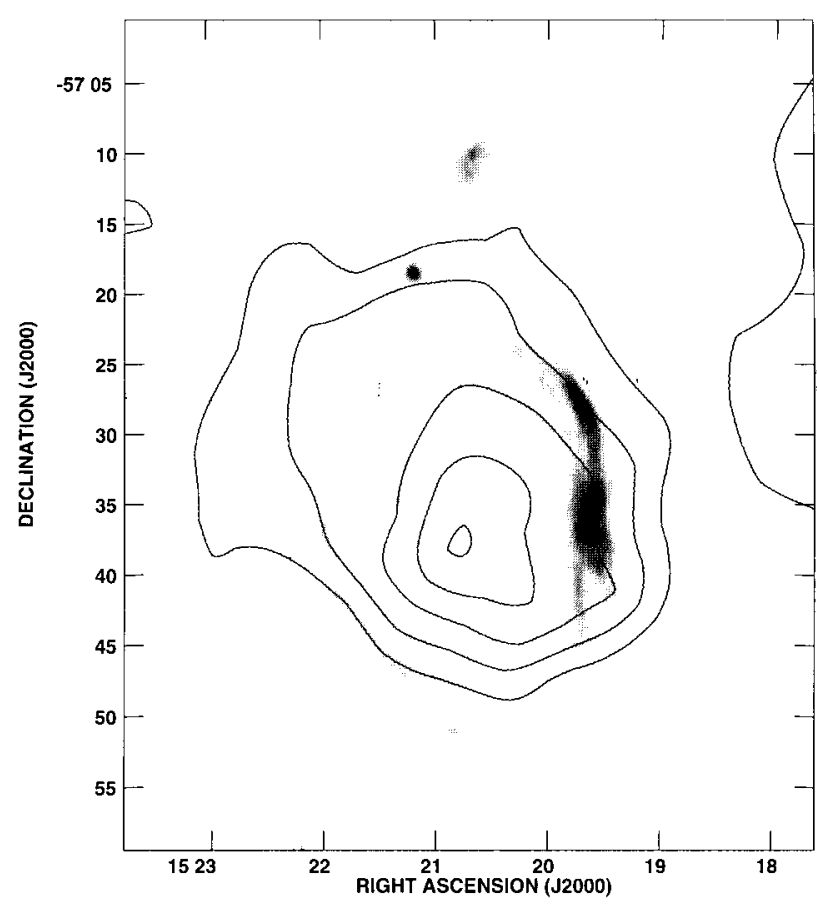

Fig. 7.- Contour map of the excess Hi emission overlaid on the $843 \mathrm{MHz}$ radio continuum image of G321.9-0.3. The radio image is from the MOST SNR catalog (Whiteoak \& Green 1996). The intensity has been integrated over $v_{\mathrm{LSR}}=$ -162 to $-109 \mathrm{~km} \mathrm{~s}^{-1}$. The contour level starts at $0.1 \mathrm{~K}$ and increases by $0.05 \mathrm{~K}$ in average antenna temperature over $v_{\mathrm{LSR}}=-162$ to $-109 \mathrm{~km} \mathrm{~s}^{-1}$.

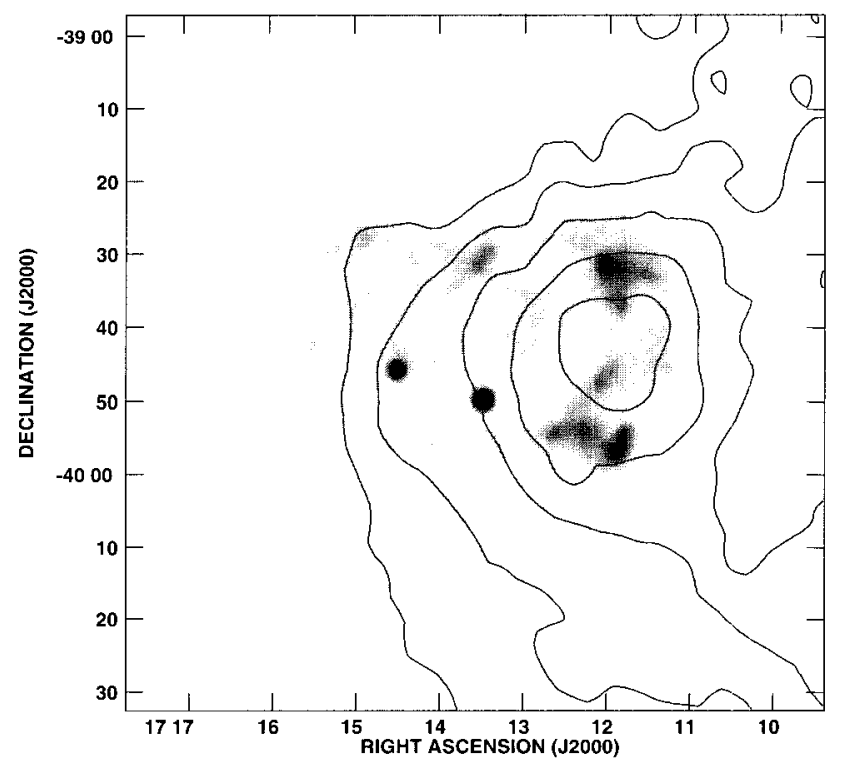

Fig. 8. - Contour map of the excess Hi emission overlaid on the ROSAT X-ray image of G347.3-0.5. The contour level starts at $0.4 \mathrm{~K}$ and increases by $0.2 \mathrm{~K}$ in average antenna temperature over $v_{\mathrm{LSR}}=68$ to $81 \mathrm{~km} \mathrm{~s}^{-1}$. 
ternatively, if the SNR is at a distance of $6 \mathrm{kpc}$ and interacting with a molecular cloud at $v_{\mathrm{LSR}}=-69 \mathrm{~km}$ $\mathrm{s}^{-1}$ along the western rim as Slane et al. (1999) suggested, then the fast-moving HI gas could have been produced by the interaction with this cloud.

\section{(d) Other Rank-3 SNRs}

G320.6-1.6 is a shell-type SNR composed of multiple arcs (Whiteoak \& Green 1996). Only the southern and eastern parts are visible, and the extent of the remnant appears to be less than a degree. Excess HI emission, which attains maxima near the northern and southern boundaries of the remnant, is apparent at positive LSR velocities, $v_{\mathrm{LSR}}=101$ to $121 \mathrm{~km} \mathrm{~s}^{-1}$. There is some confusing emission in the surrounding area, and the association is uncertain.

G332.4+0.1 (or MSH 16-51, Kes 32) is a shell-type SNR with peculiar morphology (Whiteoak \& Green 1996). The overall shape is circular with diameter of $15^{\prime}$, but the eastern shell protrudes beyond the circular boundary. From the north of the protruding structure a jet-like feature is emerging and expanding into a plume of low-brightness emission (Roger et al. 1985; Whiteoak \& Green 1996). There is a radio source at the center, but no pulsation has been discovered. Xrays have been detected toward the SNR (Brinkmann et al. 1999), but the association is not clear. There is an excess $\mathrm{HI}$ emission which attains a maximum toward the SNR. But it appears to be connected to a stronger, more extended excess emission to the northwest beyond the SNR, and the association with the SNR is uncertain.

G335.2+0.1 is a shell-type SNR of $21^{\prime}$ diameter with enhanced emission at southwest (Whiteoak \& Green 1996). A pulsar, offset from the remnant center by $\sim 4^{\prime}$, has been discovered (Kaspi et al. 1996). Its large characteristic age $\left(2.7 \times 10^{6} \mathrm{yr}\right)$, however, suggests that the association with the SNR is unlikely, although it is not impossible if the pulsar was born with a large spin period. The excess HI emission peaks in the southwest portion of the SNR where the radio continuum is enhanced. The morphological correlation is suggestive of their association.

G338.5+0.1 is a $9^{\prime}$-size SNR with amorphous morphology (Whiteoak \& Green 1996). The extent to the south is unclear because it partially overlaps with a bright HII region. Very weak excess HI emission is discovered around the SNR. The emission, however, is extended over an area larger than the SNR, and the association is not clear.

G343.1-2.3 was originally discovered by McAdam, Osborne, \& Parkinson (1993) as an SNR associated with the young $\left(\sim 1.7 \times 10^{4} \mathrm{yr}\right)$ gamma-ray pulsar PSR B1706-44 (PSR J1709-4428). But the association was questioned later based on several arguments including distance inconsistencies (Frail, Goss, \& Whiteoak 1994; Nicastro, Johnston, \& Koribalski 1996; see also Giacani et al. 2001). The SNR appears as a faint, incomplete shell with a diameter of $\sim 40^{\prime}$. There is strong $(\sim 2 \mathrm{~K})$, excess $\mathrm{HI}$ emission which attains a maximum within the western boundary of the remnant, but there is some confusing emission in the surrounding area.

G343.1-0.7 is a square-shaped, shell-type SNR with bright, $\sim 20^{\prime}$-long southern and eastern sides (Whiteoak \& Green 1996). The northern and western parts are faint and overlap with a smaller $\left(\sim 10^{\prime}\right)$ shell which is probably thermal (Whiteoak \& Green 1996). Excess $\mathrm{HI}$ emission is discovered within the western boundary of the remnant. It is superposed on a larger structure crossing western portion of the remnant, and the association is uncertain.

G350.0-2.0 is a shell-type SNR consisting of filaments with very different curvatures and brightnesses (Gaensler 1998). To the northwest it has a bright, $40^{\prime}$ long filament of small curvature, while, to southeast, it has a faint circular filament of $\sim 30^{\prime}$ diameter. The whole extent of the SNR is $\sim 45^{\prime}$. Gaensler (1998) classified it as "bilateral" SNR, and considered several mechanisms for the noncircular morphology, including multiple SN explosions and an explosion in a stratified ISM. We have detected very weak excess $\mathrm{HI}$ emission in the northwestern portion of the remnant. This is where the SNR shell is bright, and it is possible that the SNR is interacting with a dense material in this direction. The SNR shock propagating into a dense medium would be radiative, and the association of the excess HI emission with the SNR is quite possible.

\section{SUMMARY}

We have studied Hi emission lines toward 97 southern SNRs between $l=253^{\circ}-358^{\circ}$ and $|b| \lesssim 10^{\circ}$ using the SGPS data. We compare the average Hi spectra of these SNRs to those of the surrounding regions, and look for excessive emission wider than $10 \mathrm{~km} \mathrm{~s}^{-1}$ and localized at the position of the SNRs. We divide the SNRs into 3 ranks, where a higher rank means higher probability of an associated HI gas; 10 SNRs are ranked 3, 22 SNRs are ranked 2, and 65 SNRs are ranked 1. SNRs ranked 3 have HV HI gas confined to the SNR. Such gas is rare; thus, it is probable that the gas is really associated with the SNR. SNRs ranked 2 have excess H I gas confined to the position of SNRs, but not at an extreme velocity. Real association with the SNR is less certain. All other sources are ranked 1.

We present the parameters and maps of the excess emission associated with rank 2 and 3 SNRs. All rank 2 SNRs show at least some excess emission, but the following ones show particularly distinct enhancements: G286.5-1.2, G296.1-0.5, G301.4-1.0, and G302.3+0.7. All ranks 3 sources show excess emission confined to them. The ones with particularly distinct excess emission are: G272.2-3.2, G321.9-0.3, and G347.3-0.5. These three SNRs are discussed relatively in detail. The other rank 3 SNRs are briefly discussed. 
High-resolution observational studies are needed for understanding the nature of the excess $\mathrm{HI}$ emission detected in this paper. In particular, recent molecular line studies showed that HV HI gas in some SNRs are produced by the interaction with molecular clouds, e.g., W44, W51C, and IC 443 (see Koo 2003 and references therein). Therefore, both $\mathrm{HI}$ and molecular line observations are essential.

\section{ACKNOWLEDGEMENTS}

The ROSAT X-ray images in Figs. 6 and 8 were retrieved from SkyView and we acknowledge the use of NASA's SkyView facility (http://skyview.gsfc.nasa.gov) located at NASA Goddard Space Flight Center. This work was supported by S.N.U. Research Fund (334520022017).

\section{REFERENCES}

Braun, R., \& Strom, R. G. 1986, Observations of postshock neutral hydrogen in four evolved supernova remnants, A\&AS, 63, 345

Brinkmann, W., Kawai, N., Scheingraber, H., Tamura, K., \& Becker, W. 1999, ROSAT HRI observation of the supernova remnant Kes 32 and the nearby radio pulsar PSR B1610-50, A\&A, 346, 599

Case, G. L., \& Bhattacharya, D. 1998, A New Sigma -D Relation and Its Application to the Galactic Supernova Remnant Distribution, ApJ, 504, 761

Clark, D. H., Caswell, J. L., \& Green, A. J. 1975, 408 and $5000 \mathrm{MHz}$ observations of 28 new galactic supernova remnants, Australian J. PAS, 37, 1

Clark, D. H., Parkinson, J. H., \& Caswell, J. L. 1975, Is CIR X-1 a runaway binary?, Nature, 254, 674

DeNoyer, L. K. 1978, Observations of negative velocity hydrogen in IC 443, MNRAS, 183, 187

Duncan, A. R., Stewart, R. T., Campbell-Wilson, D., Haynes, R. F., Aschenbach, B., \& Jones, K. L. 1997, Radio observations of the X-ray supernova remnant G272.2-3.2, MNRAS, 289, 97

Enomoto, R. et al. 2002, The acceleration of cosmic-ray protons in the supernova remnant RX J1713.7-3946, Nature, 416, 823

Frail, D. A., Goss, W. M., \& Whiteoak, J. B. Z. 1994, The radio lifetime of supernova remnants and the distribution of pulsar velocities at birth, ApJ, 437, 781

Fukui, Y. et al. 2003, Discovery of Interacting Molecular Gas toward the TeV Gamma-Ray Peak of the SNR G 347.3-0.5, PASP 55, 61

Gaensler, B. M. 1998, The Nature of Bilateral Supernova Remnants, ApJ, 493, 781

Giacani, E. B., Frail, D. A., Goss, W. M., \& Vieytes, M. 2001, Pulsar Wind Nebulae around the Southern Pulsars PSR B1643-43 and PSR B1706-44, AJ, 121, 3133
Giovanelli, R., \& Haynes, M. P. 1979, Neutral hydrogen in supernova remnants - IC 443 and the Cygnus Loop, ApJ, 230, 404

Goss, W. M., \& Mebold, U. 1977, The distance of CIR $\mathrm{X}-1$, MNRAS, 181, 255

Green, D. A. 2001, 'A Catalogue of Galactic Supernova Remnants (2001 December version)', Mullard Radio Astronomy Observatory, Cavendish Laboratory, Cambridge, United Kingdom (available on the World-Wide-Web at "http://www.mrao.cam.ac.uk/surveys/snrs/")

Greiner, J., \& Egger, R. 1993, G272.2-3.2, IAU Circ. No. 5709

Greiner, J., Egger, R., \& Aschenbach, B. 1994, G272.23.2: a centrally filled and possibly young SNR discovered by ROSAT, A\&AL, 286, 35

Harrus, I. M., Slane, P. O., Smith, R. K., \& Hughes, J. P. 2001, New ASCA and ROSAT Study of the Supernova Remnant G272.2-3.2, ApJ, 552, 614

Kaspi, V. M., Manchester, R. N., Johnston, S., Lyne, A. G., \& Dámigo, N. 1996, Search for Radio Pulsars in Southern Supernova Remnants, AJ, 111, 2028

Kesteven, M. J., \& Caswell, J. L. 1987, Barrel-shaped supernova remnants, A\&A, 183, 118

Koo, B.-C. 2003, Shocked atomic and molecular gas in supernova remnants, ASP Conf. Ser. 289, IAU 8th Asian-Pacific Regional Meeting Proceedings Volume I, ed. S. Ikeuchi, J. Hearnshaw, \& T. Hanawa (San Francisco: ASP), 199

Koo, B.-C., \& Heiles, C. 1991, A survey of H I 21 centimeter emission lines toward supernova remnants, ApJ, 382, 204 (KH91)

Koo, B.-C., \& Heiles, C. 1995, A fast expanding H I shell in W44: A preexisting wind-blown shell overtaken by a supernova remnant, ApJ, 443, 679

Koo, B.-C., Kang, J.-h., \& McClure-Griffiths, N. 2004, HI Study of Southern Galactic Supernova Remnants, in IAU Symposium 218, Young Neutron Stars and Their Environments edited by B. Gaensler \& F. Camilo (San Grancisco:ASP), 85

Koo, B.-C., \& Moon, D.-S. 1997, Interaction between the W51C Supernova Remnant and a Molecular Cloud. I. H i 21 Centimeter Line Observations, ApJ, 475,194

Koo, B.-C., Reach, W. T., Heiles, C., Fesen, R. A., \& Shull, J. M., 1990, Detection of an expanding H I shell in the old supernova remnant CTB $80, \mathrm{ApJ}$, 364,178

Koo, B.-C., Yun, M., Ho, P. T. P., Lee, Y. 1993, Interaction between the supernova remnant CTB 80 and the ambient interstellar medium: $\mathrm{HI}$ and $\mathrm{CO}$ observations, ApJ, 417, 196

Koyama, K. et al. 1997, Discovery of Non-Thermal XRays from the Northwest Shell of the New SNR RX J1713.7-3946: The Second SN 1006?, PASJ, 49, L7 
Landecker, T. L., Roger, R. S., \& Higgs, L. A. 1980, Atomic hydrogen in a field in Cygnus $\mathrm{X}$ containing the supernova remnant G78.2+2.1, A\&AS, 39, 133

Lazendic, J. S., Slane, P. O., Gaensler, B. M., Reynolds, S. P., Plucinsky, P. P, \& Hughes, J. P. 2004, A High-Resolution Study of Nonthermal Radio and XRay Emission from Supernova Remnant G347.3-0.5, ApJL, 602, 271

Lucke, P. B. 1978, The distribution of color excesses and interstellar reddening material in the solar neighborhood, A\&A, 64, 367

McAdam, W. B., Osborne, J. L, \& Parkinson, M. L. 1993, A supernova remnant associated with the young gamma-ray pulsar PSR1706-44, Nature, 361, 516.

McClure-Griffiths, N. M., Green, A. J., Dickey, J. M., Gaensler, B. M., Haynes, R. F., \& Wieringa, M. H. 2001, The Southern Galactic Plane Survey: The Test Region, ApJ, 551, 394

Muraishi et al. 2000, Evidence for TeV gamma-ray emission from the shell type SNR RX J1713.7-3946, A\&A, 354, L57

Nicastro, L., Johnston, S., \& Koribalski, B. 1996, Evidence against the association of PSR B1706-44 with SNR G343.1-2.3., A\&A, 306, L49

Pfeffermann, E., \& Aschenbach, B. 1996, ROSAT Observation of a New Supernova Remnant in the Constellation Scorpius, Roentgenstrahlungn from the Universe, ed. H. H. Zimmermann, J. Trum̈per, \& H. Yorke (MPE Rep. 263; Garching: MPE), 267

Roger, R. S., Milne, D. K., Kesteven, M. J., Haynes, R. F., \& Wellington, K. J. 1985, A radio jet associated with the supernova remnant G332.4 + 0.1 (Kes 32), Nature, 316, 44

Slane, P., Gaensler, B. M. Dame, T. M., Hughes, J. P., Plucinsky, P. P., \& Green, A. 1999, Nonthermal XRay Emission from the Shell-Type Supernova Remnant G347.3-0.5, ApJ, 525, 357

Stewart, R. T., Caswell, J. L., Haynes, R. F., \& Nelson, G. J. 1993, Circinus X-1 - A runaway binary with curved radio jets, MNRAS, 261, 593

Uchiyama, Y., Aharonian, F. A., \& Takahashi, T. 2003, Fine-structure in the nonthermal $\mathrm{X}$-ray emission of SNR RX J1713.7-3946 revealed by Chandra, A\&A, 400,567

Wang, Z. R., Qu, Q. Y., \& Chen, Y. 1997, Is RX J1713.7-3946 the remnant of the AD393 guest star?, A\&A, 318, 59

Whiteoak, J. B. Z. \& Green, A. J. 1996, The MOST supernova remnant catalogue (MSC), A\&AS 118, 329 